\title{
Evaluation and Prediction of Driver's Bad Driving Behavior Based on the Moving Vehicle Attitude
}

\author{
Huaikun Xiang ${ }^{1}$, Shaopeng Yang ${ }^{2}$, Shi $\mathrm{An}^{2}$ and Shaoyun Ren ${ }^{1}$ \\ 1 Shenzhen Polytechnic, Shenzhen 518055, Guangdong, China \\ 2 Harbin Institute of Technology, Harbin 150001, heilongjiang, China \\ The co-first author is Shaopeng Yang \\ Correspondence should be addressed to Shaoyun Ren; \\ renshaoyun@szpt.edu.cn and \\ Huaikun Xiang; \\ xianghuaikun@szpt.edu.cn
}

\begin{abstract}
The driver takes leading part in the complex model of "human-vehicle-roadenvironment". The driving behavior has the characteristics of randomness, impulsivity and autonomy etc. which make it difficult to study the driver's driving behavior directly. Monitoring vehicle attitude is an important element of proactive safety management of public transport vehicles. This paper constructs a data acquisition system by using an InvenSense's 6-Axis inertial measurement unit (IMU) as the center of this system, which can sense the vehicle attitude information to make up for the lack of driver senses, and then builds fuzzy synthetical evaluation model which is combined with ISO 26311:1997/Amd 1:2010 standard. Finally, prediction of the driver's driving behavior in transient term has been established with Elman neural network method.
\end{abstract}

Keywords: Attitude of Vehicles, The Active Safety of Vehicles, Driving Behavior, Transient-term Prediction

\section{Introduction}

For public transport vehicles, especially buses and taxis, monitoring of vehicle attitude is an important element of proactive safety management of public transport vehicles. If the bad driving behaviors of one driver happen frequently such as the suddenly speedingup, braking and turning in the process of driving, they are not only to disrupt the traffic order, resulting in traffic accidents, but can to make the passengers feel uncomfortable, and even to cause fear psychology of riding the vehicle. In the safety analysis of road traffic system, many researchers from different countries have studied the effects of three factors on traffic accident, and found that 93\% 94\% of accidents were relative to human factors, $8 \% \sim 12 \%$ to vehicle factors, and $28 \%$ 34\% solely to roadway factors [1]. Accident studies of Mercedes-Benz for all types show: If the driver can advance one seconds to realize there is risk of accident and take appropriate corrective measures, the majority of accidents could have been avoided [2]. Therefore, achieving active monitoring for vehicle attitude is significant to regulate driver's bad driving behavior, improve the quality of public transport services and reduce the traffic accident.

The driver is the information receiver and handler in the road traffic system, and he is also the leader in the complexity model of "human-vehicle-road environment". The driver largely determines the traffic state while his driving behavior feedbacks to the transportation system directly. The study of driving behavior is a hot and difficult point in the domain of transportation. Because the driver's driving behavior has the characteristics 
of randomness, impulsivity and autonomy, it brings very great difficulty to study the driver's driving behavior directly. However, it is an effective research method to study on the driver's driving behavior indirectly by analyzing the steering vehicle attitude. Research on the steering vehicle attitude started earlier and there had practical products, such as GreenRoad company in California use their proprietary algorithms and hardware devices that can assess the real-time vehicle status based on the information of vehicle acceleration, braking, speeding and so on. In China, this field is still on the initial stage. The car attitude measuring system based on the inertial navigation and real-time differential global positioning system (GPS) has been studied in The State Key Laboratory of Vehicle Dynamic Simulation of Jilin university [3], Dr. Zhang et al in Tsinghua university design the algorithm to identify and evaluate the driver's bad driving behavior using driving behavior model of hierarchical structure [4], Jiaoyan Yang et al in Chinese Academy of Sciences examine the effects of personality variables on Chinese drivers' unsafe driving behaviors and accident involvement from the point of view of Psychology [5], their results are not conducive to the popularization and application. At present most studies of car attitude more stay in judging vehicle attitude such as the vehicle acceleration, deceleration, turning posture, etc., and difficult to determine further the bad driving behavior such as a sharp turn, the snap acceleration, and deceleration, etc.

Based on the short-term prediction research above, when a dangerous situation occurs, the driver can receive an early warning from the system, which can avoid the sudden operation of the driver in case of an emergency reaction, so corrective action can be carried out smoothly and improve vehicle safety. In daily life, the sudden operation of the driver tends to cause more accidents, but such accidents can be reduced $80.7 \%$ by early warning [6].

In this paper a data acquisition system by using InvenSense's 6-Axis IMU (3-Axis Gyro and 3-Axis Accelerate) as the center of this system has been constructed, which can sense the vehicle attitude information to make up for the lack of driver's senses (shown in Fig. 1), and then builds fuzzy evaluation model which is combined with ISO 26311:1997/Amd 1:2010 [7] standard to assess the automobile bad driving behavior by using this information. Finally prediction of the attitude of the vehicle driving has been established with Elman neural network for further research.

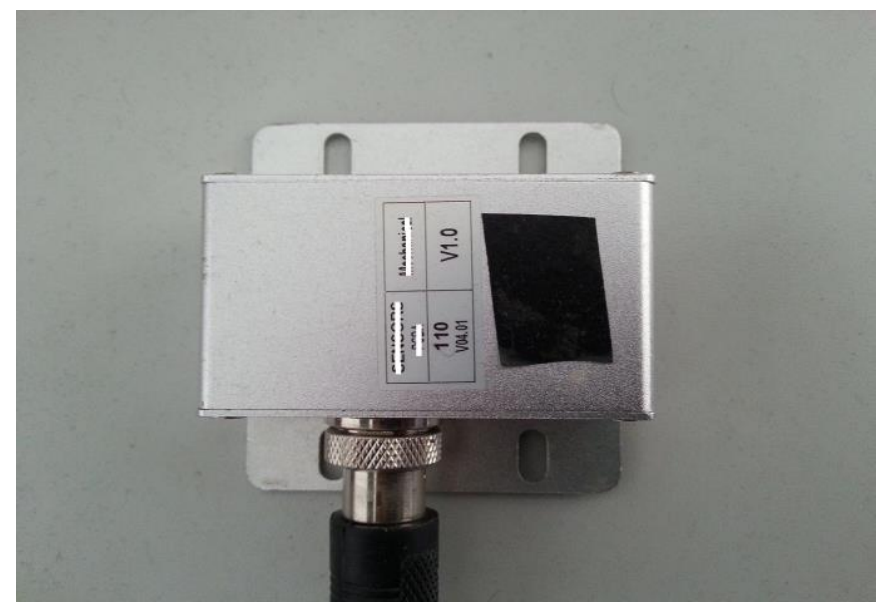

Figure 1. The 6-Axis Inertial Measurement Unit (IMU)

\section{The Evaluation of the Driver's Bad Driving Behavior}

It is different that different driving experience, mind, mood and temperament determine the driver and passengers' perception of the automobile running status. With the brake as an example, according to the sensory information of the human body obtained, some think it was a sudden braking, but some people might think that was slowly braking. 
Therefore, the demarcation of judge whether the brake behavior is a bad driving behavior is uncertain and fuzzy. If we provide merely a clear boundaries to determine this behavior, it is bound to introduce the person's subjective factors (including observation and judgment errors), which may cause false or omit to the test results.

In this paper, it is studied that how to use statistical knowledge to analyze and make data fusion between the person's subjective experience and objective parameters, and to evaluate the driver's bad driving behavior based on the synthetical evaluation of the vehicle attitude.

\subsection{The Establishment of ISO 2631-1:1997/AMD 1:2010 Standard Algorithms}

ISO 2631-1:1997/Amd 1:2010 headed "Mechanical vibration and shock-Evaluation of human exposure to whole-body vibration" [7], quantifies vibration exposure limit of human bearing during the process from a solid surface to the human body in the $1 \sim 80 \mathrm{~Hz}$ frequency range, and provides the comfort of the human body under different vibration. Three axis acceleration values are commonly used as the measured values in algorithm of vehicle vibration measurement. Practice has proved that with three axes of acceleration as the basis for evaluation of vibration to access the degree of vehicle vibration i effective [8]. Concrete steps in this algorithm are as follows:

1. For the vibration signal (three-axis acceleration), discrete Fourier transform (DFT) transforms it into the frequency domain, and the conversion formula can be described as:

$$
\mathrm{X}(f)=\sum_{n=0}^{N-1} x(n) e^{-j \frac{2 \pi}{N} t}
$$

Where $X(\mathrm{n})$ is a finite vibration signal with the length number $\mathrm{N}$ in the time domain, and $X(\mathrm{n})$ is the three-axis acceleration in this paper, and $X(f)$ is a vibration signal in the frequency domain.

2. To compute the root mean square (RMS) value of one-third octave and weight acceleration at the centre of one-third octave. Formula of computing the mean square error can be used:

Formula of computing the root mean square (RMS) value of one-third octave

$$
a_{i}=\sqrt{\frac{1}{f_{i u}-f_{i l}} \int_{f_{i l}}^{f_{i u}} X^{2}(f) d f} \quad i=1,2,3 \ldots \ldots .20
$$

Where $a_{i}$ is the root mean square (RMS) value of one-third octave, its unit is $m / s^{2}, f_{i u}$ is an upper cut-off frequency on the $i^{t h}$ frequency band, $f_{i l}$ is an lower cutoff frequency on the $i^{\text {th }}$ frequency band, and $X(f)$ is a frequency domain signal value of an acceleration. The results of Equation (2) will be used in Equation (3).

Because the human body has different reaction under the different frequency vibration indifferent directions, if in the center of the frequency given a weighting factor, it is able to make a real measured data which may response the feelings of human body. ISO26311 (1997) /AMD 1:2010 gives a table about the center frequency of one-third octave which corresponds to the weighted factor of each axis. Thus, by look-up table, the equation (3) can be used to weight acceleration of each axis. 


$$
a_{w j}=\sqrt{\sum_{i=1}^{20}\left(k_{i} a_{i}\right)^{2}}, \quad i=1,2, \ldots \ldots .20
$$

Where $a_{w j}$ is a weighted acceleration of each axis, its unit is $\mathrm{m} / \mathrm{s}^{2}$, and $j=x, y, z$,and $k_{i}$ is a weighted coefficient in the $i^{\text {th }}$ one-third octave band.

According to the random input running test method of automobiles provided by the China national standard GB/T 4970-1996, total acceleration of each axis are weighted that the weights of $\mathrm{X}$-axis and $\mathrm{Y}$-axis are 1.4 and that of $\mathrm{Z}$-axis is 1.0. Equation (4) can be used to calculate the RMS value of the total acceleration.

$a_{w}=\sqrt{\left(1.4 a_{w x}\right)^{2}+\left(1.4 a_{w y}\right)^{2}+a_{w z}^{2}}$

Where $a_{w}$ is a RMS value of the total acceleration, its unit is $\mathrm{m} / \mathrm{s}^{2}$, and $a_{w x}$ 、 $a_{w y} 、 a_{w z}$ is the RMS value of each axis by the Equation (3)

3. Based on the RMS value of the total acceleration and subjective feeling of the human's body, the judgment of comfort can be shown in Table 1 .

Table 1. The RMS Value of the Total Acceleration and Subjective Feeling of the Human's Body

\begin{tabular}{cc}
\hline The RMS of the total acceleration $a_{w}\left(m / s^{2}\right)$ & subjective feeling \\
& \\
\hline $0.315 \sim 0.63$ & Not uncomfortable \\
& A little uncomfortable \\
$0.5 \sim 1.0$ & Fairly uncomfortable \\
$0.8 \sim 1.6$ & Uncomfortable \\
$1.25 \sim 2.5$ & Very uncomfortable \\
$>2.0$ & Extremely comfortable \\
\end{tabular}

\subsection{Fuzzy Synthetic Evaluation Model}

Driver's bad driving behavior occurs not only with his or her random and impulsive, autonomy characteristics, and also relevant to other different features of the driver such as the gender, age, driving experience, character, etc., so the fuzzy synthetical evaluation model is established in this paper. Fuzzy synthetical evaluation method is a synthetical assessment method that applies fuzzy mathematical principles to evaluate things and phenomenon affected by variety of factors [9]. It is a kind of combination with qualitative and quantitative, accurate and precise method of analysis and evaluation. Fuzzy synthetic evaluation model is composed of factor set $\mathrm{U}$, evaluation set $\mathrm{V}$ and judgment matrix R.Its implementation steps are as follows:

1. Build the factors set, that is $U=\left\{u_{1}, u_{2}, \ldots \ldots . . u_{n}\right\}$.

2. Build the evaluation set $V=\left\{v_{1}, v_{2}, \ldots v_{m}\right\}$.

It's a set composed of $m$ kinds of evaluation standards.

3. Establish the single-factor evaluation matrix $R_{i}$ from $\mathrm{U}$ to $\mathrm{V}$. 


$$
R=\left(r_{i j}\right)_{m n}=\left[\begin{array}{cccc}
r_{11} & r_{12} & \ldots & r_{1 m} \\
r_{21} & r_{22} & \ldots & r_{2 m} \\
\ldots & \ldots & \ldots & \ldots \\
r_{n 1} & r_{n 2} & \ldots & r_{n m}
\end{array}\right]
$$

Here $r_{i j} \in[0,1]$ means the degree of the evaluation objects to each $v_{i}$ on each $u_{i}, i=1,2, \ldots, n, j=1,2, \ldots, m$.

In the evaluation of the current information system, this matrix $\mathrm{R}$ is determined mostly by the following method [10]: An estimation group made up of several experts' judges and determines what level of each single factor belongs to, which is based on their experience and professional knowledge. The meaning of $r_{i j}$ is the percentage of the experts who determine single factor $u_{m}$ belongs to $v_{j}$ level.

4. Determine the evaluation factors with weight vector A.

It is very important to choose the appropriate weight set. The importance degree of the factor should be given during the synthetic evaluation, which is namely the relative weight between the indices and layers.

$$
A=\left\{a_{1}, a_{2}, \ldots a_{n}\right\}
$$

Where $a_{i}$ is the weight of the $i^{\text {th }}$ factor, and $a_{1}+a_{2}+\ldots+a_{n}=1\left(0 \leq a_{i} \leq 1\right)$.

5. Get the results of evaluation.

The results of evaluation can be gotten by multiplying the vector of the index weight and the matrix $\mathrm{R}$ of single-factor evaluation:

$\mathrm{B}=\mathrm{A} \circ \mathrm{B}=\left(b_{1}, b_{2}, \ldots b_{m}\right)$

Based on a large number of experiments and questionnaire survey, the application of fuzzy synthetic evaluation model may be used as follows: Build the factors set $U=\left\{u_{1}, u_{2}, u_{3}, u_{4}\right\}=\{$ the driver's gender, driver's age, the driver's driving experience, the driver's character $\}$, and passengers (including experienced skilled drivers, experts and follow-up of passengers) subjective feeling by bus constitute the evaluation set $V=\{$ not uncomfortable, a little uncomfortable ,fairly uncomfortable, uncomfortable, very uncomfortable, extremely comfortable \}, and evaluate the driver's bad driving behavior by the estimation group to get evaluation matrix R. We checked the evaluation results of the B with that of the ISO 26311:1997/Amd 1:2010 standard algorithm, and then did revision, so that this combination of subjective evaluation and objective evaluation to determine the fuzzy synthetical evaluation model could avoid mistakes of evaluation.

\section{The Moving Vehicle Attitude Prediction by Elman Neural Network}

\subsection{The Learning Algorithm of Elman Neural Network}

Elman neural network (Elman NN) was proposed by Jeffrey L. Elman in 1990. Unlike static feedforward neural networks, Elman network is a dynamic recurrent neural network. This network is obtained by another feedback loop from the output of hidden layer to the input of this layer, which constitutes the "context layer" that retains information between 
observations [11]. This network type consists of an input layer, a hidden layer, an output layer and a context layer. Typical structure of Elman neural network is depicted in figure 2. The input layer and output layer respectively play signal transmission and linear weighting roles in the network. The transfer function of hidden layer can be linear or nonlinear. The context layer without weighting is fed from the output layer.

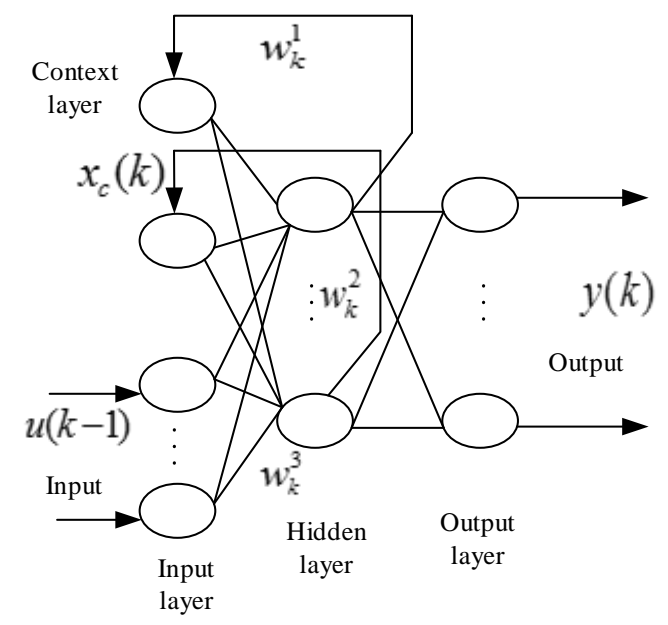

Figure 2. The Structure of Elman Neural Network

This allows the context layer can remember the hidden layer's output values of previous moments, and makes context layer sensitive to the historical data, which increases network's own ability to handle dynamic information. Figure 2, as an example, the nonlinear state space expression of Elman network is:

$$
\begin{aligned}
& x(k)=f\left[w_{k}^{1} x_{c}(k)+w_{k}^{2} u(k-1)\right] \\
& x_{c}(k)=x(k-1) \\
& y(k)=g\left[w_{k}^{3} x(k)\right]
\end{aligned}
$$

Here, $u(k-1)$ is the external input, $x(k)$ is the output of hidden layer and $y(k)$ is the output of the network. $w_{k}^{1}, w_{k}^{2}$ and $w_{k}^{3}$ is the matrix of connection weights, which is respectively expressed from the context layer to the hidden layer, from the input layer to the hidden layer and from the hidden layer to the output layer. $f$ and $g$ are translation functions of the hidden layer and the output layer.

The back propagation (BP) learning algorithm is used in the learning of Elman neural network to modify weights. The network was trained with gradient descent method. So that through the network weights of each layer to adjust, it may make sample output and identify the output to minimize the mean square error (MSE). Specific learning algorithm is:

$$
\begin{aligned}
& E=\sum_{k=1}^{n}[y(k)-d(k)]^{2} \\
& x(k)=f\left[w_{k}^{1} x_{c}(k)+w_{k}^{2} u(k-1)\right]
\end{aligned}
$$

Where $d(k)$ is the desired output.

Elman neural network learning algorithm flow chart is shown in Figure 3. 


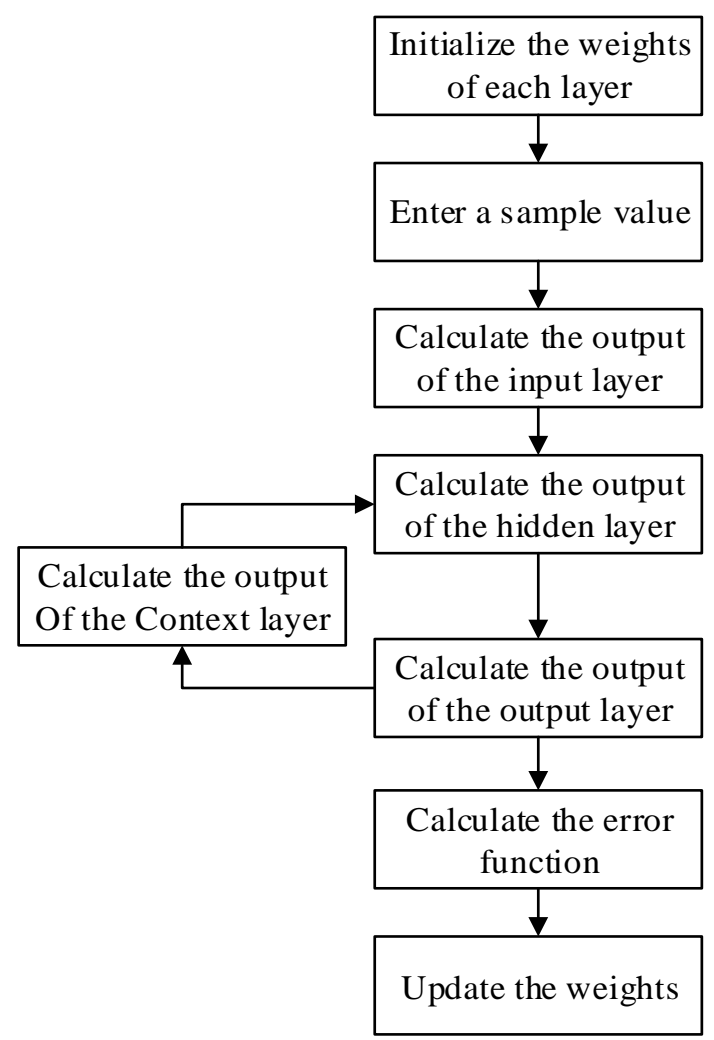

Figure 3. Flow Chart of the Elman Neural Network Learning Algorithm

\subsection{The Application of Elman Neural Network in Moving Vehicle Attitude Prediction}

With strong ability to learn, Elman neural network model seeks rules of time series data which is historical data during the process of driving and then predicts the attitude of the car. This algorithm to predict vehicle steering attitude does not need to consider the driver's characteristics, information such as road surface power spectrum and wind resistance coefficient.

We assume that taking the vehicle attitude angles of the first $\mathrm{N}$ points to predict the next. The mapping function can be expressed as follows:

$$
x_{n}=f\left(x_{n-1}, x_{n-2}, x_{n-N}\right)
$$

We have the other preparation work to do, which are described as follows:

Firstly, construct sample set. The data given vehicle attitude angle is to be divided into training samples and testing samples. The sample set is constructed by using cyclic prediction rules that take the value of previous forecast as the next input, as shown in Table 1.

\begin{tabular}{cc}
\hline Variable values of input & Target values of output \\
\hline$\left(x_{1}, x_{2}, \ldots, x_{N-1}\right)$ & $x_{N}$ \\
$\left(x_{2}, x_{3}, \ldots, x_{N}\right)$ & $x_{N+1}$ \\
$\ldots$ & $\ldots$ \\
\hline
\end{tabular}


Secondly, normalize data. Data normalization is designed to avoid numeric overflow occurs during a computer simulation of neural networks, thus ensuring the performance of neural networks and its stability [12]. Neural network input values are normalized by using Equation (13), which can be converted into values falling in the interval [-1, 1]; Neural network output values are renormalized by using Equation (14), which can be converted into normal values.

$$
\begin{aligned}
& \hat{\mathrm{L}}=\frac{2 \mathrm{~L}-\mathrm{L}_{\text {max }}-L_{\text {min }}}{\mathrm{L}_{\text {max }}-L_{\text {min }}} \\
& L=\frac{1}{2}\left[\left(\mathrm{~L}_{\text {max }}-L_{\text {min }}\right) \hat{\mathrm{L}}+\mathrm{L}_{\text {max }}+L_{\text {min }}\right]
\end{aligned}
$$

Where $\mathrm{L}_{\max }$ and $L_{\min }$ are respectively the maximum value and minimum value in the sample set.

Finally, establish Elman neural network. This paper set the input layer has 6 neurons and the output layer has 1 neurons. According to [13], the numerical relationship between the input layer neurons and the hidden layer neurons is approximately as follows:

$$
N_{2}=2 N_{1}+1
$$

Where $N_{1}$ the number of input is layer neurons and $N_{2}$ is the number of hidden layer neurons, $N_{2}=13$ in this paper.

\subsection{Prediction Results and Analysis}

\section{Calculating the Attitude Angle}

In order to collect data and test the algorithm designed above, it is set up that the Data acquisition system and equipment on Volkswagen Santana (as shown in figure 4) in this paper. Sampling frequencies of the IMU is $10 \mathrm{HZ}$. This paper takes 900 historical data as sample data, as shown in Figure 5. Data from the first to the 510th is selected as training samples, and the other ones from the 511th to the 900th are selected the testing samples.

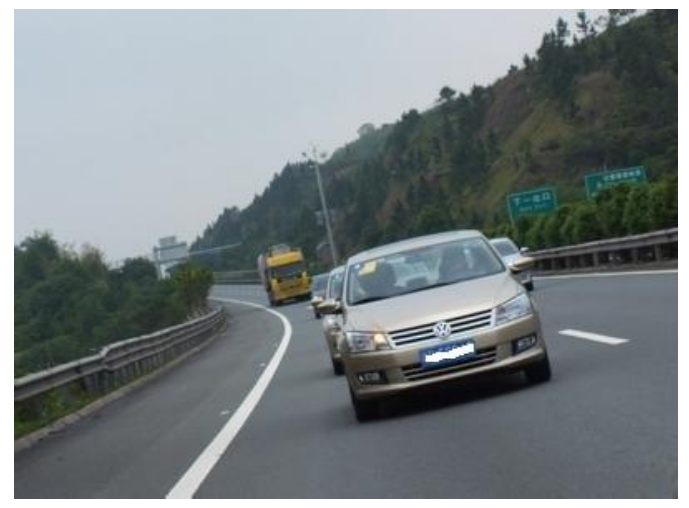

(a)

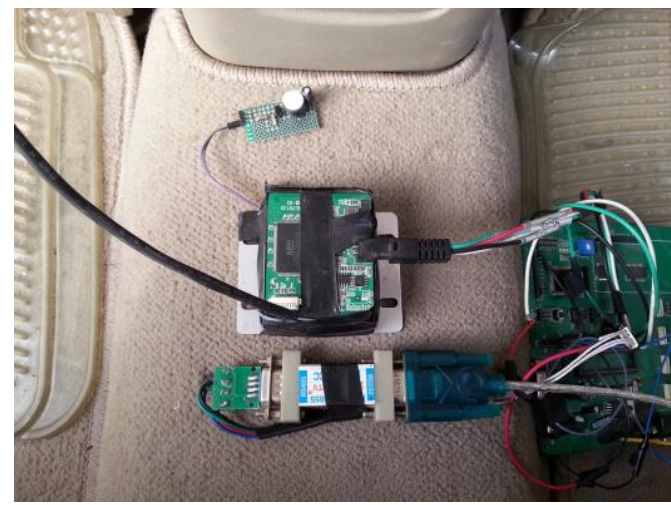

(b)

Figure 4. Data Acquisition System and Equipment on Volkswagen Santana

Using quaternion method calculates the attitude matrix, and then the result is shown in Figure 6. 


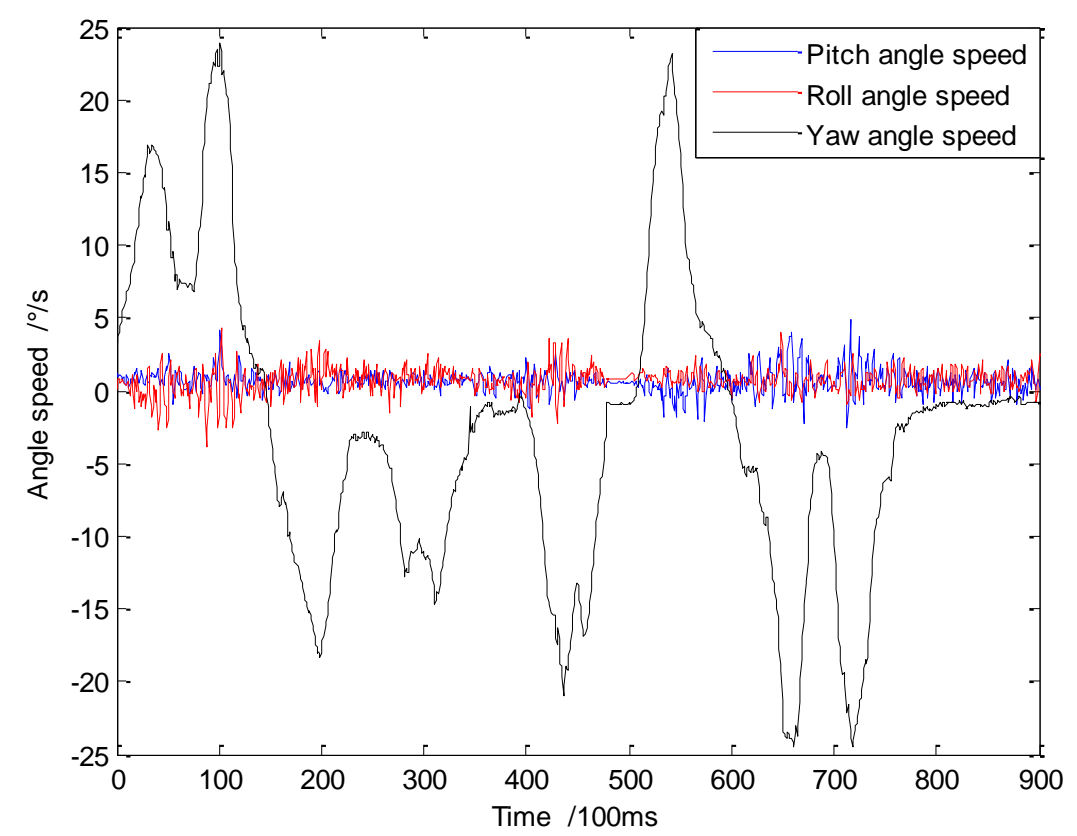

Figure 5. Three-Axis Angular Velocity Diagram of the Car
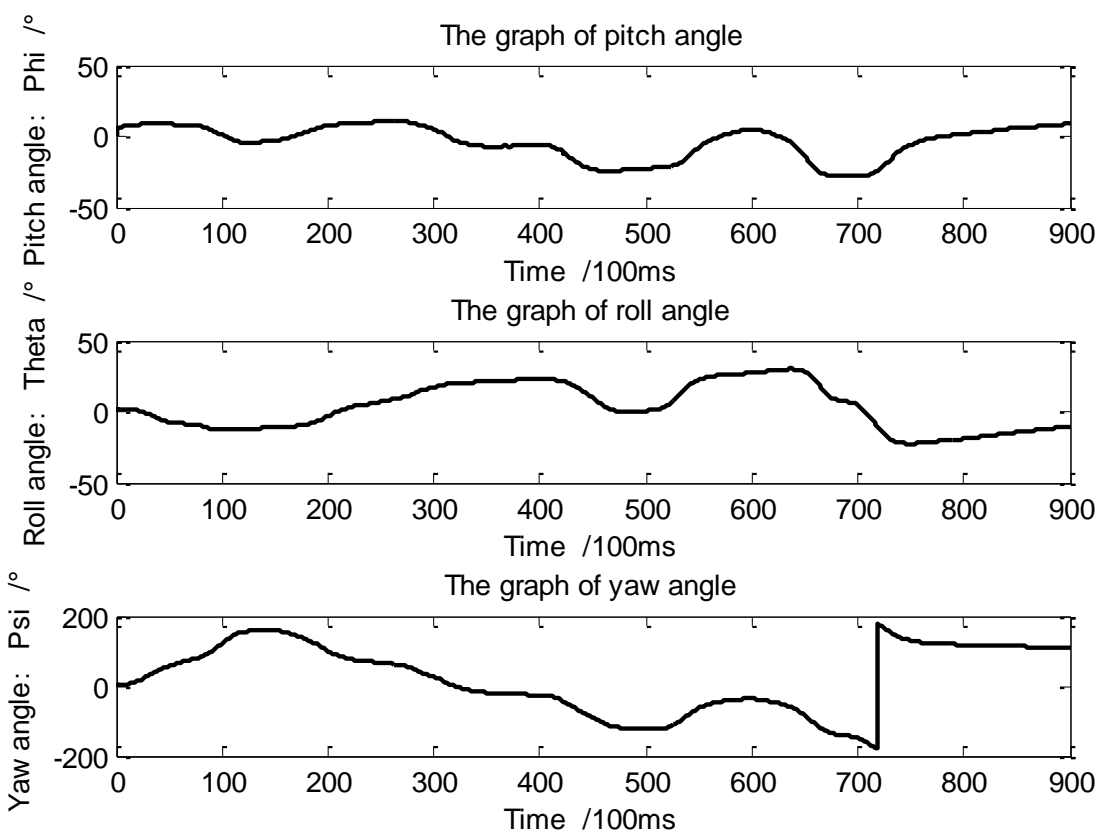

Figure 6. The Attitude Angle Change Curve of the Car

\section{The Raining and Testing of Elman Neural Network}

In the research of vehicle rollover warning, roll angle is an important indicator of rollover. This paper focuses on the roll angle as the example, and carries on the forecast to the attitude of vehicle. For the same sample, it is used that the BP neural network (BPNN) prediction in this paper, and its result is compared with the result of Elman neural network. The trained each of the two models are used to predict the roll angle for the test samples. Firstly, we do one step prediction which is also to predict the posture in advance 0.1 seconds, and its result is shown in Figure 7. The results of one seconds posture 
prediction which are compared with the test samples are shown in figure 8 . That of two seconds posture prediction is shown in Figure 9.

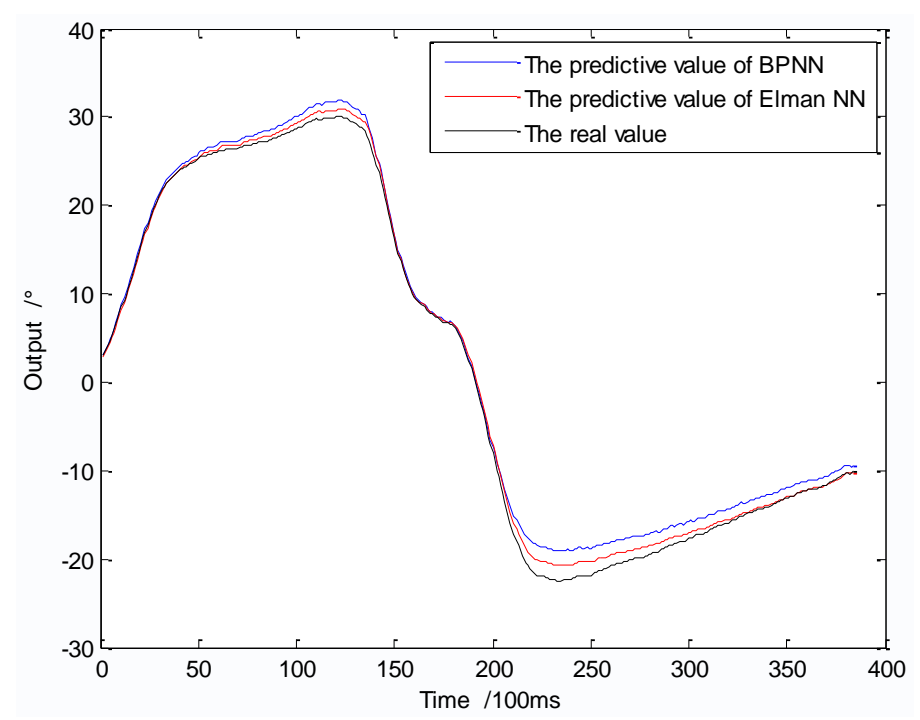

Figure 7. The Results of 0.1 Seconds Posture Prediction

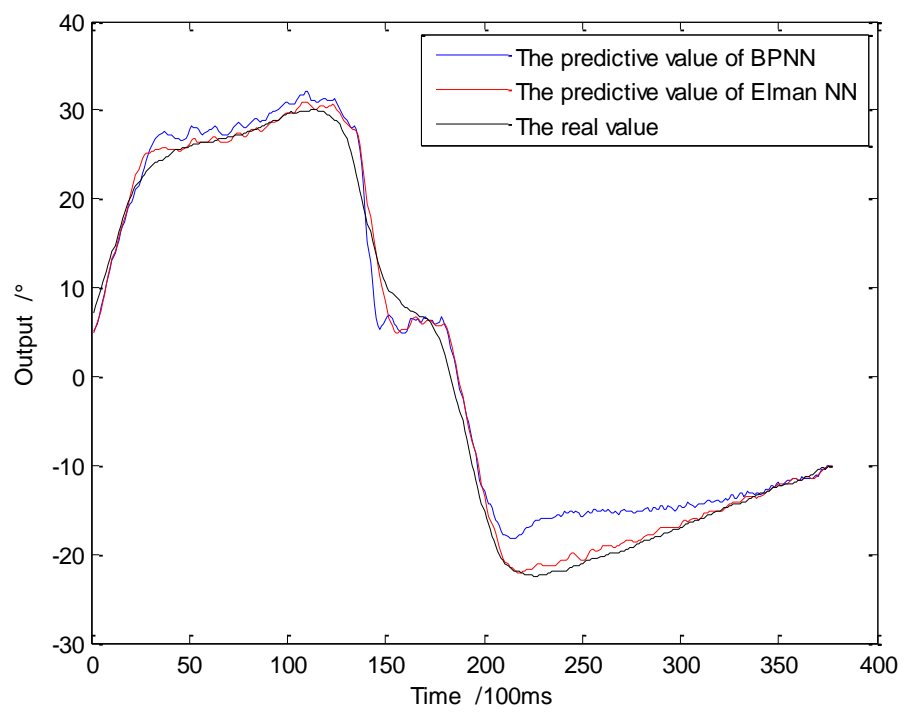

Figure 8. The Results of 1 Seconds Posture Prediction 


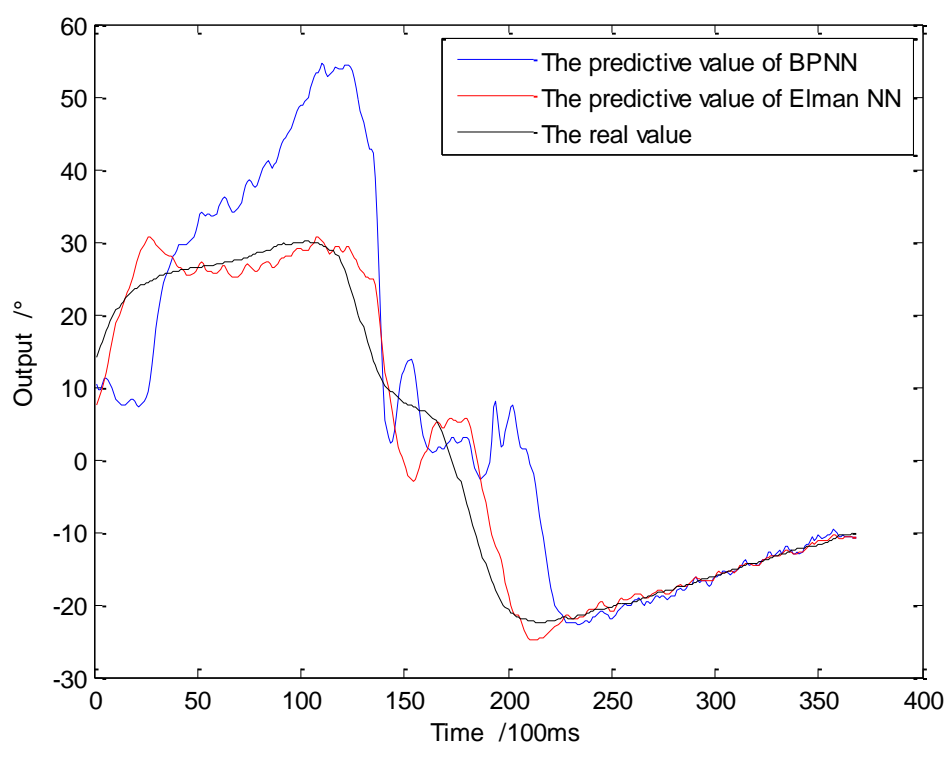

Figure 9. The Results of 2 Seconds Posture Prediction

In order to test the prediction effect, we use the indexes of mean absolute error (MAE), mean square error (MSE) and root mean squared error (RMSE) to evaluate the prediction performance. The expressions of them are defined as follows:

$$
\begin{aligned}
& \text { MAE }=\frac{1}{n} \sum_{i=1}^{n}\left(A_{t}-F_{t}\right) \\
& M S E=\frac{1}{\mathrm{n}} \sum_{t=1}^{n}\left(A_{t}-F_{t}\right)^{2} \\
& R M S E=\sqrt{\frac{1}{\mathrm{n}} \sum_{t=1}^{n}\left(A_{t}-F_{t}\right)^{2}}
\end{aligned}
$$

\begin{tabular}{|c|c|c|c|c|c|c|c|c|c|}
\hline & \multicolumn{3}{|c|}{$\begin{array}{l}\text { The errors of } 0.1 \\
\text { seconds prediction }\end{array}$} & \multicolumn{3}{|c|}{$\begin{array}{c}\text { The errors of } 1 \\
\text { seconds prediction }\end{array}$} & \multicolumn{3}{|c|}{$\begin{array}{c}\text { The errors of } 2 \\
\text { seconds prediction }\end{array}$} \\
\hline & MAE & $\begin{array}{c}\mathrm{MS} \\
\mathrm{E}\end{array}$ & $\begin{array}{c}\text { RMS } \\
\text { E }\end{array}$ & MAE & $\begin{array}{c}\mathrm{MS} \\
\mathrm{E}\end{array}$ & $\begin{array}{c}\text { RMS } \\
\text { E }\end{array}$ & MAE & MSE & $\begin{array}{c}\text { RMS } \\
\text { E }\end{array}$ \\
\hline Elman NN & 0.60 & 0.20 & 0.45 & 0.99 & 0.71 & 0.84 & 2.29 & 4.72 & 2.17 \\
\hline BPNN & 1.36 & 0.91 & 0.95 & 2.34 & 2.94 & 1.71 & 7.90 & $\begin{array}{c}47.4 \\
0\end{array}$ & 6.89 \\
\hline
\end{tabular}

Where $A_{t}$ is the predicted value and $F_{t}$ is the actual value.

Table 3. For the Errors of Prediction

The comparison of prediction errors of the two methods is shown as follows:

Table 4. Comparison of Elman Neural Network and BP Neural Network

\begin{tabular}{cccccc}
\hline Model & Network structure & $\begin{array}{c}\text { Learning } \\
\text { algorithm }\end{array}$ & $\begin{array}{c}\text { Number of } \\
\text { training }\end{array}$ & Precision \\
\hline Elman NN & $6,13,1$ & BP & 500 & 0.01 \\
BP NN & $6,13,1$ & BP & 210 & 0.01 \\
\hline
\end{tabular}


The charts from Figure 7, to Figure 9, indicate that the increase in the number of the time in advance to predict vehicle roll has a significant impact on the rapid increase in the number of the prediction errors. Meanwhile, as which can be seen from the table 3, the prediction errors of BP neural network are far higher than that of Elman neural network. In addition, when the fluctuation of vehicle roll angle is large, Elman neural network can better track the changes, especially it can be seen from figure 9 . The table 4 indicates that under the condition of the network structure and learning is the same, BP neural network need fewer training to achieve the accuracy of reservation than BP neural network, which can save the training time, but when the two trained neural network are used to test the testing sample, the errors of BPNN prediction are higher, which can be seen in table 3 .

\section{Conclusions}

In the paper, firstly, a data acquisition system has been built based an InvenSense's 6Axis inertial measurement unit (IMU), and compute the car attitude matrix by using the fourth Runge-Kutta method. Secondly, fuzzy synthetic evaluation model has been developed to assess the bad driving behavior in combine with ISO 2631-1:1997/Amd 1:2010 standard and to avoid the randomness, subjective uncertainty and fuzziness of knowledge in the process of evaluation. Finally, Elman network was applied in prediction of car attitude, and by comparing the BP neural network, Elman network has better effects in the transient-term prediction of the moving car posture. Research results in the paper has realized the prediction of the car posture ahead of 1 second and 2 seconds, and can evaluate the driver's behavior in advance and restrain the bad driving behaviors.

\section{Acknowledgements}

Funding for the research on the driver's bad driving behavior and its trend prediction was provided by the Open Foundation of Shenzhen Key Laboratory of Urban Planning and Decision Making (Grant UPDMHITSZ2014B06). We also appreciate all those experts who provided advice in the process of research.

\section{References}

[1] Z. Guo, S. Fang, Editor, Road safety engineering, People's traffic press, Beijing, (2003), pp.70-71.

[2] Shanyong Chen, Xiyue Huang, Wenzhong Zhang. Intelligent vehicle collision avoidance system based on DSP research. Automation technology and application. vol. 7, no. 1, (2005).

[3] X. Guan, D. Yan, Z. Gao, "Based inertial navigation and real-time differential GPS vehicle motion state testing system”, Jilin University (Engineering Science), vol. 36, no. 1 (2006), pp.15-19.

[4] W. Zhang, W. Wang, etc., "Bad driver driving behavior recognition method", 2011 14th Automotive Safety Technology Conference Proceedings, Beijing: China Automotive Engineering Society of Automotive Safety Technology Branch, (2012), pp. 208-212.

[5] J. Yang, F. Du, W. Qu, Gong Z, X. Sun. "Effects of Personality on Risky Driving Behavior and Accident Involvement for Chinese Drivers", Traffic Injury Prevention. vol. 14, no. 4 (2013), pp. 565-571.

[6] G. Leen and D. Heffernan, Expanding automotive electronic systems, IEEE Computer.. vol. 35, no. 1(2002), pp. 88-93.

[7] International Organization for Standardization,ISO 2631:1997/Amd 1:2010 Mechanical vibration and shock-Evaluation of human exposure to whole-body vibration-Part 1:General requirements,ISO(1997)

[8] E. Edwan, J. Zhang, J. Zhou and O. Loffeld, Reduced DCM based attitude estimation using low-cost IMU and magnetometer triad, in Proceedings of the 2011 Workshop on Positioning Navigation and Communication (2011), pp. 1-6.

[9] G. Schmidt, R. Phillips .Schmidt, G., Phillips, R., INS/GPS integration architectures. NATO RTO Lecture Series RTO-EN-SET-116 (2011), pp. 1-16.

[10] Tianguang Zhang, Xiuping Wang, Lixia Wang. Strapdown inertial navigation technology. Beijing: national defense industry press (2010), pp.70-71.

[11] J. L. Liu, Q. X. Li, and Y. H. Wang, "Risk analysis in ultra-deep scientific drilling project-A fuzzy synthetic evaluation approach", International Journal of Project Management. vol. 31, no. 3, March (2013), pp. 449-458. 
[12] J. Zhourun, L. Zhang. "Fuzzy neural network design and fuzzy TECH of MATLAB based”,Beijing: Electronic Industry Press (2010), pp.110-111.

[13] C.H Lin, "Recurrent modified Elman neural network control of PM synchronous generator system using wind turbine emulator of PM synchronous servo motor drive", International Journal of Electrical Power \& Energy Systems. 52 (2013), pp.143-160.

\section{Authors}

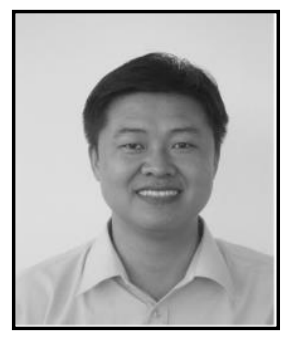

Huaikun Xiang, male, Ph.D., associate professor, director of professional, graduated from Beijing university of technology, just now is working in the School of Automotive \& Transportation Engineering, Shenzhen Polytechnic, Shenzhen, Guangdong, China. Main research areas of interest are the methods of the automotive active safety monitoring and analysis, the intelligent traffic signal control technology. So far, Mr. XIANG has been hosted a total of 15 scientific research projects and published 25 academic papers as the first author.

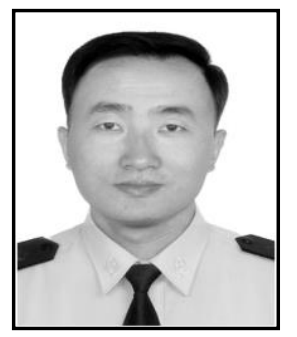

Shaopeng Yang, 45 years old, graduated with a Bachelor Degree, now studying for On-job Doctorate. I'm working in Nanshan Traffic Police Station of Shenzhen Public Security Bureau Traffic Police Department as the Deputy Director, engaged in transportation engineering. With rich experience, I expert in solving the problem of traffic congestion and had earned a fruitful achievement of 5 patents. 
International Journal of Security and Its Applications

Vol.9, No.11 (2015) 